\title{
TYPICAL CONTINUOUS FUNCTIONS ARE NOT CHAOTIC IN THE SENSE OF DEVANEY
}

\begin{abstract}
We show that typical continuous functions of the form $f: M \rightarrow M$, where $M$ is a compact metric space with the fixed-point property and the absolute-retract property, are not chaotic in the sense of Devaney. Typical continuous functions on the compact interval have been shown to be chaotic in terms of other definitions of chaos. Results are also presented concerning the chain recurrent set for typical continuous functions and concerning functions for which the chain recurrent set is the entire space.
\end{abstract}

\section{Definitions and Preliminaries}

For general background and notation, we refer the reader to [1] and [2]. In this paper we shall refer to certain known results in the monograph of Block and Coppel [2] by chapter and number, and ask the reader to consult the bibliography of the monograph for information on the original papers. We let $f: M \rightarrow M$ represent a continuous function on a compact metric space $M$, with metric $d$, where $M$ has the fixed-point property, and where $M$ has the absolute-retract property. No additional properties are put on $M$, unless otherwise indicated. We say that a compact metric space has the absoluteretract property if every closed ball in $M$ is a retract of $M$. Compact $n$-cells in $\mathbb{R}^{n}$ are examples of spaces which have the fixed-point property and the absolute-retract property. We recall that every retract of a space with the fixed-point property also has the fixed-point property.

Key Words: asymptotically stable set, chain recurrent set, Devaney's chaos, sensitive dependence on initial conditions, transitivity, typical continuous functions.

Mathematical Reviews subject classification: 26A18, 54H20.

Received by the editors October 30,1999

*The author would like to thank the referee for valuable remarks and suggestions. 
We let $C$ denote the set of all continuous functions of the form $f: M \rightarrow M$. By "typical continuous functions", we mean all functions in some residual subset $S$ of $C$, where the complement of the residual set $S$ is the countable union of closed nowhere dense sets. If we equip $C$ with the sup metric $D(f, g)=\sup \{d(f(x), g(x)): x \in M\}$, then $C$ is a complete metric space, and our residual subset $S$ is dense in $C$.

The iterates of a function $f: M \rightarrow M$ are defined inductively such that $f\left(f^{n}(x)\right)=f^{n+1}(x)$, where $f^{n}$ is the $n$-fold composition of $f$. The trajectory of $x$ in $M$ is the sequence $\{f(x)\}_{n=0}^{\infty}$, where $f^{0}(x)=x$. The orbit of $f$ at $x$, denoted by $\gamma(x, f)$, is the point set $\left\{f^{n}(x): n \geq 0\right\}$. The omega-limit set of $f$ at $x$, denoted by $\omega(x, f)$, is the set of all subsequential limit points of the orbit. We denote the closure of a set $A$ by $\mathrm{Cl}(A)$. A continuous function on a compact interval will be denoted by $f: I \rightarrow I$, where $f$ has no additional properties unless otherwise indicated. We shall denote the set of all continuous functions on a compact interval by $C(I, I)$.

A function $f: M \rightarrow M$ is transitive if for every pair of non-empty open sets $U$ and $V$ in $M$, there is a positive integer $k$ such that $f^{k}(U) \cap V \neq \emptyset$. A function $f: M \rightarrow M$ is transitive iff there is a point $x$ in $M$ such that $\omega(x, f)=M$. [2]. There are many examples in the literature of transitive functions, including the tent map on the unit interval and the logistic function (sometimes called the population equation) $h(x)=r x(1-x)$ for $r=4$. A function $f: M \rightarrow M$ has sensitive dependence on initial conditions if there exists $\delta>0$ such that for any $x$ in $M$, and for any open set $U$ containing $x$, there exists $y$ in $U$ and a positive integer $n$ such that $d\left(f^{n}(x), f^{n}(y)\right)>\delta$. In Devaney's definition of chaos [7], a function $f: X \rightarrow X$, where $X$ is an arbitrary compact metric space, is chaotic if $f$ has sensitive dependence on initial conditions, has a dense set of periodic points, and is transitive.

\section{Devaney's Chaos and Chain Recurrence}

Theorem 1. Let $M$ be any compact metric space with fixed-point property, and the absolute-retract property. Let $C$ denote the set of all continuous functions of the form $f: M \rightarrow M$. Then there is a residual subset $S$ in $C$ such that every function in $S$ is not transitive.

Proof. Since $M$ is a compact metric space, there is a countable dense subset $D \subset M$. For each point $x$ in $D$, and for each rational distance $\gamma>0$, we define an open ball $B(x, \gamma)$ with center $x$ and radius $\gamma$. Since there are countably many such open balls, we may enumerate them. Let $B_{i}$ be any such open ball. 
For each $B_{i}$ we define the set $S_{i}$ to be the set

$\left\{f \in C\right.$ :either $f$ has no fixed point in $\mathrm{Cl}\left(B_{i}\right)$; or if $f$ has a fixed point in $\mathrm{Cl}\left(B_{i}\right)$, then there is an open ball $U_{i} \subset \mathrm{Cl}\left(B_{i}\right)$ such that $\left.f\left(\mathrm{Cl}\left(U_{i}\right)\right) \subset U_{i}\right\}$.

We first show that $S_{i}$ is an open subset of $C$. Let $\epsilon>0$ be given. Let $g$ be any function in $S_{i}$. There are two cases:

CASE 1: Suppose that $g$ has a fixed point in $\mathrm{Cl}\left(B_{i}\right)$. Since $g \in S_{i}$, we have $g\left(\mathrm{Cl}\left(U_{i}\right)\right) \subset U_{i}$ for some open ball $U_{i} \subset \mathrm{Cl}\left(B_{i}\right)$. Since $g\left(\mathrm{Cl}\left(U_{i}\right)\right)$ is compact, there is a positive distance $\delta>0$ between the compact set $g\left(\mathrm{Cl}\left(U_{i}\right)\right)$ and the boundary of $U_{i}$. Then for any continuous function $h \in C$ such that $D(g, h)<\min \{\delta / 2, \epsilon\}, h\left(\mathrm{Cl}\left(U_{i}\right)\right) \subset U_{i}$. Also, since $M$ has the fixed-point property and the absolute-retract property, $h$ has a fixed point in $\mathrm{Cl}\left(B_{i}\right)$. Then $h$ is an element of $S_{i}$.

Case 2: Suppose $g$ has no fixed point in $\mathrm{Cl}\left(B_{i}\right)$. Since $g(x) \neq x$ for any $x$ in $\mathrm{Cl}\left(B_{i}\right)$, there is a positive distance between $g(x)$ and $x$ for each $x$ in $\mathrm{Cl}\left(B_{i}\right)$. Since $\operatorname{Cl}\left(B_{i}\right)$ is compact, we may let $\delta=\min \left\{d(g(x), x): x \in \mathrm{Cl}\left(B_{i}\right)\right\}$. Let $h$ be any function in $C$ such that $D(g, h)<\min \{\delta / 2, \epsilon\}$. Then for any $x$ in $\mathrm{Cl}\left(B_{i}\right)$,

$$
d(g(x), x) \leq d(g(x), h(x))+d(h(x), x),
$$

which implies that

$$
d(g(x), x)-d(g(x), h(x)) \leq d(h(x), x) .
$$

Since $d(g(x), x)$ is greater than or equal to $\delta$, and since $d(g(x), h(x))<\delta / 2$, this implies that $\delta-\delta / 2=\delta / 2 \leq d(h(x), x)$. It follows that $h$ does not have a fixed point in $\mathrm{Cl}\left(B_{i}\right)$. Hence $S_{i}$ is open in $C$.

To show that $S_{i}$ is dense in $C$, let $f$ be any element in $C$. We show that there is a function $h$ in $S_{i}$, arbitrarily close to $f$ in $C$. Let $\epsilon>0$ be given. Either $f$ has a fixed point in $\mathrm{Cl}\left(B_{i}\right)$ or it does not. Suppose that $f$ has a fixed point in $\mathrm{Cl}\left(B_{i}\right)$. Let $x_{o}$ be the fixed point of $f$ in $\mathrm{Cl}\left(B_{i}\right)$. We construct the function $h$ so that $h\left(x_{o}\right)=x_{o}$; that is, $h$ also has a fixed point at $x_{o}$. At all points in the closed ball $\mathrm{Cl}\left(B\left(x_{o}, \epsilon / 2\right)\right)$, we make $h$ constant, so that $h(x)=x_{o}$ for all $x$ in $\operatorname{Cl}\left(B\left(x_{o}, \epsilon / 2\right)\right)$. At all other points of the domain of $h$, we only require that $h$ be $\epsilon$-close to $f$. Then we may assume that $h$ is continuous and $h$ is contained in an $\epsilon$-neighborhood of $f$. Let $U_{i}^{*}$ be the open ball which is centered at $x_{o}$ and has radius $\epsilon / 4$. Since $x_{o}$ could sit in the boundary of $\mathrm{Cl}\left(B_{i}\right)$, we define $U_{i}$ to be $U_{i}^{*} \cap \mathrm{Cl}\left(B_{i}\right)$. Then we have that $h\left(\mathrm{Cl}\left(U_{i}\right)\right) \subset U_{i}$. It follows that $h$ is in $S_{i}$. Suppose that $f$ does not have a fixed point in $\mathrm{Cl}\left(B_{i}\right)$. Then we proceed as in Case 2 above to show that there is a function $h$ in $S_{i}$, 
arbitrarily close to $f$, such that $h$ does not have a fixed point in $\mathrm{Cl}\left(B_{i}\right)$. Hence, $S_{i}$ is dense in $C$. Since $S_{i}$ was chosen arbitrarily, we conclude that every $S_{i}$ is open and dense in $C$.

Let $S=\cap_{i=1}^{\infty} S_{i}$. Then $S$ is residual in $C$. Now let $g$ be any element of $S$. Since $g$ has a fixed point in $M$, for some open ball $U$ in $M, g(\mathrm{Cl}(U)) \subset U$. Hence, since $U \cap M$ is a proper subset of $M$, no iterate $g^{k}(U)$ of $U$ meets any other nonempty open set which is disjoint from $U$, and $g$ is not transitive on $M$.

Remark 2. We remark that in [1] it is shown that typical continuous functions of the form $f: I \rightarrow I$ are not transitive, and a related result was obtained in [10] for measure-preserving homeomorphisms of the closed unit square.

Corollary 3. There is a residual subset $S$ of the set $C$ of all continuous functions of the form $f: M \rightarrow M$ such that all functions in $S$ are not chaotic in the sense of Devaney.

Remark 4. An excellent survey of various definitions of chaos is contained in [8]. In contrast to the result in Corollary 3, in [6] and [9] it is shown that functions which are chaotic in the sense of $\mathrm{Li}$ and Yorke ( $\mathrm{L}-\mathrm{Y}$ chaos) are residual in $C(I, I)$. In [2] it is shown that functions which are chaotic in the sense of Block and Coppel (B-C chaos) comprise an open subset of $C(I, I)$. It follows that the set of functions which are not B-C chaotic cannot be residual in $C(I, I)$, again in contrast to the result in Corollary 3 above. In [5] Bruckner and Ceder introduce a notion of chaos which is strictly intermediate between B-C chaos and L-Y chaos; they show that a continuous function $f: I \rightarrow I$ is chaotic iff it has the property that all of its infinite omega-limit sets are perfect.

Corollary 5. There is a residual subset $E$ of $C(I, I)$ such that every function in $E$ is Li-Yorke chaotic but is not chaotic in the sense of Devaney.

Proof. The intersection of two residual subsets is again residual.

Let $f: X \rightarrow X$, where $X$ is a compact metric space. Following [2], we say that a non-empty closed set $A \subset X$ is Lyapunov-stable if for each open set $U$ containing $A$, there exists an open set $V$ containing $A$ such that $\gamma(x, f) \subset U$ for every $x$ in $V$. The set $A$ is asymptotically-stable if it is Lyapunov-stable and, in addition, there exists an open set $U_{o}$ containing $A$ such that $\omega(x, f) \subset A$ for every $x \in U_{o}$. Asymptotically stable sets are sometimes called "attractors".

Theorem 6. There is a residual subset $S$ of the set $C$ of all continuous functions of the form $g: M \rightarrow M$ such that all functions in $S$ have an asymptotically-stable set. 
Proof. This follows immediately from the fact that the typical function $g$ in the proof of Theorem 1 above has an inward set. That is, since $g(\mathrm{Cl}(U)) \subset U$, we consider the set $A=\cap_{n \geq 0} g^{n}(\mathrm{Cl}(U))$. For each $n, g^{n}(\mathrm{Cl}(U))$ is nonempty and closed, and $g^{n}(\mathrm{Cl}(U)) \supset g^{n+1}(\mathrm{Cl}(U))$. Then since $M$ is compact, the set $A$ is nonempty, and by $[2, \mathrm{~V} .13], A$ is asymptotically stable.

Corollary 7 deals with the concept of chain recurrence. Let $f: M \rightarrow M$ and let $x$ and $y$ be points in $M$ such that $x \neq y$. An $\epsilon$-chain from $x$ to $y$ is a finite sequence of points $\left\{x_{0}, x_{1}, \ldots, x_{n}\right\}$, where

$$
x=x_{0} \text { and } y=x_{n} \text { and } d\left(f\left(x_{i-1}\right), x_{i}\right)<\epsilon \text { for } i=0,1, \ldots, n .
$$

If for any $\epsilon>0$ there exists an $\epsilon$-chain from $x$ to $y$, then we say that $x$ can be chained to $y$. If $x$ can be chained to itself, then $x$ is a chain recurrent point. The set of all chain recurrent points is called the chain recurrent set. We denote the chain recurrent set of a function $f$ by $C R(f)$.

Corollary 7. There is a residual subset $S$ of the set $C$ of all continuous functions of the form $f: M \rightarrow M$, where $M$ is also connected, such that for every function $f$ in $S, C R(f) \neq M$.

Proof. See Lemma 1 of [3] and apply Theorem 6.

For the next result, we let $C(X, X)$ denote the set of continuous functions of the form $f: X \rightarrow X$ with the sup metric, where $X$ is a compact metric space. The lemma shows that if any two functions in $C(X, X)$ are sufficiently close, then their $n$th iterates remain close.

Lemma 8. Let $f$ and $g$ be elements of $C(X, X)$. Then for any positive integer $n$, and for any $\epsilon>0$, there exists $\delta>0$ such that if $D(f, g)<\delta$, then $D\left(f^{n}, g^{n}\right)<\epsilon$.

Proof. First we show that the lemma is true for $k=1$. That is, for any $\epsilon>0$, there exists $\delta>0$ such that if $D\left(f^{1}, g^{1}\right)=D(f, g)<\delta$, then $D\left(f^{2}, g^{2}\right)<\epsilon$. Let $\epsilon>0$ be given. Since $f(X)$ and $g(X)$ are compact, by the uniform continuity of $f$, choose $\delta>0$ such that if $d(f(x), g(x))<\delta$, then

$$
d(f(f(x)), f(g(x)))<\frac{\epsilon}{2} \text { for all } x \in X .
$$

Let $D(f, g)<\min \{\delta, \epsilon / 2\}$. Then for all $x \in X$,

$$
\begin{aligned}
& d\left(f^{2}(x), g^{2}(x)\right)=d(f(f(x)), g(g(x))) \\
& \leq d(f(f(x)), f(g(x)))+d(f(g(x)), g(g(x)))<\frac{\epsilon}{2}+\frac{\epsilon}{2}=\epsilon .
\end{aligned}
$$


It follows that $D\left(f^{2}, g^{2}\right)<\epsilon$. Hence, the lemma is true for $k=1$. Since $f^{k}$ and $g^{k}$ are continuous for any nonnegative integer $k$, the argument is the same for any positive integer $k>1$. This proves the second part of the induction argument.

Remark 9. Although Lemma 8 is exactly the result we need to prove Theorem 10 below, the result in Lemma 8 can be strengthened as follows:

Let $f$ and $g$ be elements of $C(X, X)$. Then for any positive integer $n$, and for any $\epsilon>0$, there exists $\delta>0$ such that if $D(f, g)<\delta$, then $D\left(f^{k}, g^{k}\right)<\epsilon$ for $k=1,2, \ldots, n$.

For the proof, since Lemma 8 provides a $\delta_{k}>0$ such that $D(f, g)<\delta_{k}$ implies that $D\left(f^{k}, g^{k}\right)<\epsilon$, choose $\delta=\min \left\{\delta_{k}: k=1,2, \ldots, n\right\}$.

For the result in Theorem 10, we let $f: Y \rightarrow Y$ be a continuous function on a compact metric space $Y$, where $Y$ is locally connected and connected, and where $Y$ has the fixed-point property and the absolute retract property. By Theorem $\mathrm{C}$ of [4], the chain recurrent set is not the whole space $Y$ (i.e., $C R(f) \neq Y$ ) iff there exists a connected open set $U$ with $U \neq Y$ and $f^{n}(\mathrm{Cl}(U)) \subset U$ for some positive integer $n$. We denote by $C(Y, Y)$ the set of all continuous functions of the form $f: Y \rightarrow Y$.

Theorem 10. Let $C(Y, Y)$ be the set of continuous functions of the form $f: Y \rightarrow Y$, where $Y$ is a compact metric space which is locally connected and connected. Suppose further that $Y$ also has the fixed-point property and the absolute retract property. Then the set of functions in $C(Y, Y)$ which have every point chain recurrent, is nowhere dense in $C(Y, Y)$.

Proof. By Corollary 7, there is a residual subset $D$ of $C(Y, Y)$ such that for each function $g$ in $D, C R(g) \neq Y$. For any such $g$ in $C(Y, Y)$, by the result in [4], there is a connected open set $U$ with $U \neq Y$ and $g^{n}(\mathrm{Cl}(U)) \subset U$ for some positive integer $n$. Since $g^{n}(\mathrm{Cl}(U))$ is compact, there is a positive distance $\delta>0$ between $g^{n}(\mathrm{Cl}(U))$ and the boundary of $U$. By Lemma 8, we can find $\epsilon>0$ such that for any function $h$ such that $D(g, h)<\epsilon$ then $D\left(g^{n}, h^{n}\right)<\delta / 2$, and $h^{n}(\mathrm{Cl}(U)) \subset U$. Then, again by the result in [4], $C R(h) \neq Y$. Since any function $h$, sufficiently close to $g$, has the property that $C R(h) \neq Y$, we conclude that the set of functions in $C(Y, Y)$ with every point chain recurrent, is nowhere dense in $C(Y, Y)$.

\section{References}

[1] S. J. Agronsky, A. M. Bruckner, and M. Laczkovich, Dynamics of typical continuous functions, J. London Math. Soc. 40 (1988), no. 2, 227-243. 
[2] L. S. Block and W. A. Coppel, Dynamics in one dimension, Lect. Notes in Math., vol. 1513, Springer-Verlag, 1992.

[3] L. S. Block and E. M. Coven, Maps of the interval with every point chain recurrent, Proc. Amer. Math., 98 (1986), no. 3, 513-515.

[4] L. S. Block and J. E. Franke, The chain recurrent set, attractors, and explosions, Ergod. Th.\&Dynam. Sys., 5 (1985), 321-327.

[5] A. M. Bruckner and J. Ceder, Chaos in terms of the map $x \rightarrow \omega(x, f)$, Pac. J. Math. 56 (1992), no. 1, 63-96.

[6] G. J. Butler and G. Pianigiani, Periodic points and chaotic functions in the unit interval, Bull. Austral. Math. Soc. 18 (1978), no. 2, 255-265.

[7] R. Devaney, Introduction to chaotic dynamical systems, Benjamin Cummings, Menlo Park, CA, 1986.

[8] S. H. Jones, Applications of the Baire category theorem, Real Analysis Exchange 23 (1997/98), no. 2, 363-394.

[9] P. E. Kloeden, Chaotic difference equations are dense, Bull. Austral. Math. Soc. 15 (1976), 371-379.

[10] J. C. Oxtoby, Measure and Category, 2nd ed., Springer-Verlag, Heidelberg, 1980. 
R. A. Mimna 